\title{
Valor Social Integrado (VSI) del Hospital de Santa Marina en Bilbao: monetizando el valor social para los 'stakeholders'
}

\section{Frumen Molinero}

Departamento de Salud, Gobierno Vasco

\section{Leire San-José}

Universidad del País Vasco/Euskal Herriko Unibertsitatea (UPV/EHU)

leire.sanjose@ehu.eus

\author{
Álvaro Molinero \\ Health Business Consultant, Everis
}

Gaur egun, erakundeek haien burua legitimatzeko joera dute sortu ohi duten balio sozialagatik; ez soilik ekonomikoagatik. Osasunaren kasuan, ikuspegi hori agerikoa da, osasun-entitateek haien gaixoei eta ongizateari zor baitiote. Horretarako, interesdunek zein balio sozial lortzen duten adierazi beharko dute. Artikulu honetan harago joaten gara eta balio sozialak ezagutzeaz beste, aldagai ekonomikoen bitartez ere adierazi egingo dira. Horrela, ospitale batek, Bilboko Santa Marina Ospitaleak, sortzen duen balio soziala zein den jakin daiteke. Horrez gain, bere aplikazioan alderdi zailenak zeintzuk diren, zer ekarpen egin zien balio soziala islatzeak eta beste ospitale batzuetara nola hedatu daitekeen aztertzen da artikulu honetan, lana bereziki aberasten duen alderdia.

\section{GAKO-HITZAK:}

Giza balioa, eragina, stakeholder, balio-sorrera, osasuna, efizientzia.
En la actualidad, las organizaciones tienden a legitimarse por el valor social que generan, y no solo por el económico. Para el caso de la sanidad, esta perspectiva es obvia, ya que las entidades sanitarias se deben a sus pacientes y a su bienestar. Para ello, será necesario saber qué opinan los stakeholders perceptores del valor social. Este artículo va más allá, y no solo se conocen los valores sociales, sino que se llegan a monetizar. Así se logra saber cuál es el valor social generado por un hospital, el Hospital de Santa Marina de Bilbao. Además, se analiza cuáles son los aspectos más complicados en su aplicación, qué les aportó y cómo es posible extenderlo a otros hospitales.

\section{Palabras clave:}

Valor social, impacto, stakeholders, creación de valor, sanidad, eficiencia. 


\section{Introducción}

Hoy en día las empresas se preocupan cada vez más por el valor social que generan y no solo por el resultado económico (Harrison et al., 2020), por lo que el aporte social ha dejado de estar en un segundo plano y podríamos decir que estamos ante un cambio donde las empresas quieren medir la función social que aportan a su entorno, en vez de preocuparse solamente de generar y logar beneficios económicos. Por ello, actualmente cada vez es más importante apreciarlo y evaluarlo, bien para la reputación e imagen de la empresa, bien para establecer mejores comunicaciones con los diferentes órganos y entidades sociales y públicas, 0 bien para mejorar la estrategia empresarial hacia la creación de valor social.

Este trabajo tiene por objeto demostrar que es posible monetizar el valor social de un hospital mediante una contabilidad social y el logro de su valor social integrado (VSI en adelante). Seguiremos el modelo de contabilidad social desarrollada hace ya diez años por Retolaza et al. (2016), que ha sido aplicado en varios ámbitos, como lo son el de la economía social (Lazkano y Beraza, 2019; Lazkano y San-José, 2020), el de centros especiales de empleo (Retolaza et al., 2014), el de la hostelería (Guzmán et al., 2020) o el de universidades (Ayuso et al., 2020). En este trabajo exponemos un nuevo ámbito, los centros sanitarios, y en concreto se aplica la contabilidad social a un hospital. Las razones para aplicar la contabilidad social en vez de el SROI, han sido fundamentalmente tres. En primer lugar, la contabilidad social es más idónea para analizar situaciones pasadas. En segundo lugar, una contabilidad social holística es más completa y global. Y, en tercer lugar, permite la construcción de ratios más allá del propio SROI. Así, con una contabilidad enfocada a lo social, como ocurre con la tradicional será posible obtener no solo el SROI, sino también otras ratios, como por ejemplo el SVAI (véase San-José et al., 2019).

Para contextualizar, se debe señalar que desde que en 1983 se aprobara la ley de Osakidetza, el sistema sanitario público vasco se ha convertido en un organismo autónomo, financiado con presupuesto público, para prestar atención sanitaria integral a los ciudadanos y con el objetivo de formar un único sistema asistencial con todos los recursos públicos existentes. Este sistema sanitario público vasco está integrado por dos organizaciones, que son el Departamento de Salud y Osakidetza, y están presentes en los tres territorios históricos de la Comunidad Autónoma del País Vasco. El
${ }^{1}$ También se encarga de: la evaluación de los niveles de la salud de la población, las condiciones de acceso a los servicios sanitarios, establecer prioridades de política sanitaria, ampliar el catálogo de prestaciones básicas ofrecidas por el sistema nacional de salud y asumir las responsabilidades en lo que se refiere a derechos y participación ciudadana (Osakidetza, 2016). financiar el sistema sanitario público, mientras que Osakidetza es un ente público con plena capacidad que se dedica a facilitar servicios sanitarios mediante las organizaciones sanitarias públicas.

Las organizaciones sanitarias integradas (OSI) son el elemento facilitador que permite agrupar centros y hospitales de referencia según su demarcación en la geografía vasca. Así, en Euskadi hay 13 OSI, (5 en Bizkaia, 6 en Gipuzkoa y 2 en Araba), 2 hospitales de crónicos (Santa Marina y Gorliz) y tres redes de salud mental (Araba, Bizkaia y Gipuzkoa). Además, existen tres organizaciones cuyo ámbito territorial se extiende a toda Euskadi: Centro Vasco de Transfusión y Tejidos Humanos, Emergencias y Osatek. Esta estructura se completa con la dirección general, que es la estructura directiva para el control, coordinación estratégica y apoyo en la gestión de las anteriores organizaciones de servicios.

Esta nueva organización de los centros de Osakidetza nace de la necesidad de dar una respuesta a las nuevas necesidades de la población, una población cada vez más envejecida. La población de 650 más años podría aumentar en más de 110.000 personas hasta 2026, por lo que su peso llegará al 26,5\% -casi 7 puntos más que en 2013-. De este total, 32.000 personas irían al grupo de más de 85 años, que pasará a suponer el 4,6\% del total (Eustat, 2014).

La esperanza de vida para 2026 se prevé que llegue hasta los 82,6 años para los hombres y 88,4 para las mujeres, mientras que en 2010 se situaba en los 79,1 años para los hombres y 85,6 años para las mujeres (Eustat, 2014). Por lo tanto, continúa el proceso de envejecimiento de la población, haciendo que cada vez más personas y de edad más avanzada necesiten atención sanitaria y sociosanitaria. En este trabajo demostramos que la monetización del valor social es una dinámica útil y eficiente en los hospitales; es posible realizarla, pero es posible trasladarla más allá y aplicarla en diferentes hospitales. Para ello, en los siguientes apartados exponemos, en primer lugar, el caso objeto de análisis, el Hospital de Santa Marina de Bilbao, para posteriormente establecer cuáles han sido los pasos para exponer el valor social generado por el hospital. En un siguiente apartado se abordarán las reflexiones y utilidades de esta aplicación. Finalizaremos con las conclusiones, limitaciones y futuras líneas de investigación.

\section{Análisis de caso: Hospital de Santa Marina}

En 1930 se procedió a construir el edificio Victoria Eugenia en la ladera del monte Artxanda en Bilbao, que serviría como base para la construcción del Complejo Sanatorial Santa Marina. Fue entonces cuando ingresó el primer paciente. Desde sus comienzos, el hospital atendía a personas con enfermedades respiratorias (sobre todo enfermos de tuberculosis). Nueve años después, se inauguró el edificio Briñas, que ayudó a pasar de 100 a 250 camas. 
Con el objetivo de proporcionar atención sanitaria a los niños, en 1942 construyeron el edificio Víctor Tapia, con 100 camas. Poco después, en 1944, se inauguró el actual pabellón, denominando al conjunto Grupo Sanatorial Santa Marina, que contaba con 750 camas. El complejo llego a tener 850 camas en 1958. En el año 1979 se procedió al cierre definitivo de los pabellones Briñas y Tapia, visto que bajó el número de enfermos de tuberculosis y, un año después, el hospital paso a las manos del Gobierno Vasco. Desde entonces, el hospital ha experimentado un cambio y ha pasado de atender a enfermos principalmente con patología respiratoria a atender a pacientes crónicos.

\subsection{Misión}

La misión del Hospital de Santa Marina consiste en ser el hospital de crónicos de referencia en la atención integral de personas, principalmente mayores, en fases avanzadas de sus enfermedades y al final de sus vidas. Trabaja de forma coordinada con otras organizaciones para garantizar su continuidad asistencial y el regreso al entorno más adecuado a sus necesidades.

\subsection{Visión}

En 2020 desea ser reconocido como un hospital monográfico de crónicos:

- Que da respuesta de calidad a las necesidades de las personas, atendiendo al reto del envejecimiento, la cronicidad y la dependencia.

- Que presta una atención humanizada, cercana, de confianza y adecuada a pacientes y familiares a través de la implicación de sus profesionales.
- Orientado a conseguir que los cuidados asistenciales sean los cuidados intensivos del trato y del confort.

\subsection{Pacientes}

En la Figura 1 se puede analizar la distribución de tipo de pacientes a partir del trabajo para estratificar el riesgo de pacientes desarrollado por kaiserpermanente.org en Estados Unidos.

La mayor parte de los pacientes del hospital son personas de edad avanzada, con patología crónica compleja, con quienes hay que trabajar desde la doble vertiente de la cura y el cuidado. Muchas de ellas son personas que muestran un nivel de fragilidad importante, ya que ante procesos intercurrentes se pueden enfrentar a una situación de pérdida de independencia, lo cual obliga a trabajar $y$ atender sus necesidades de un modo coordinado con otros profesionales del sistema de salud y de los servicios sociales.

Un fenómeno creciente derivado del aumento de la esperanza de vida es la presencia de personas de edad avanzada con un aceptable nivel de salud y que, tras un episodio agudo, pueden ver modificada sustancialmente su nivel de autonomía. El Hospital de Santa Marina ofrece los recursos adecuados para la atención de este tipo de pacientes, debido a la experiencia acumulada, la especialización de sus profesionales y los servicios disponibles.

Finalmente, un colectivo importante para el hospital son los pacientes con necesidades de cuidados paliativos, a quienes destina una atención personalizada y específica dentro de la red.

Figura 1. Kaiser Permanente adaptada a las características de los pacientes del Hospital de Santa Marina

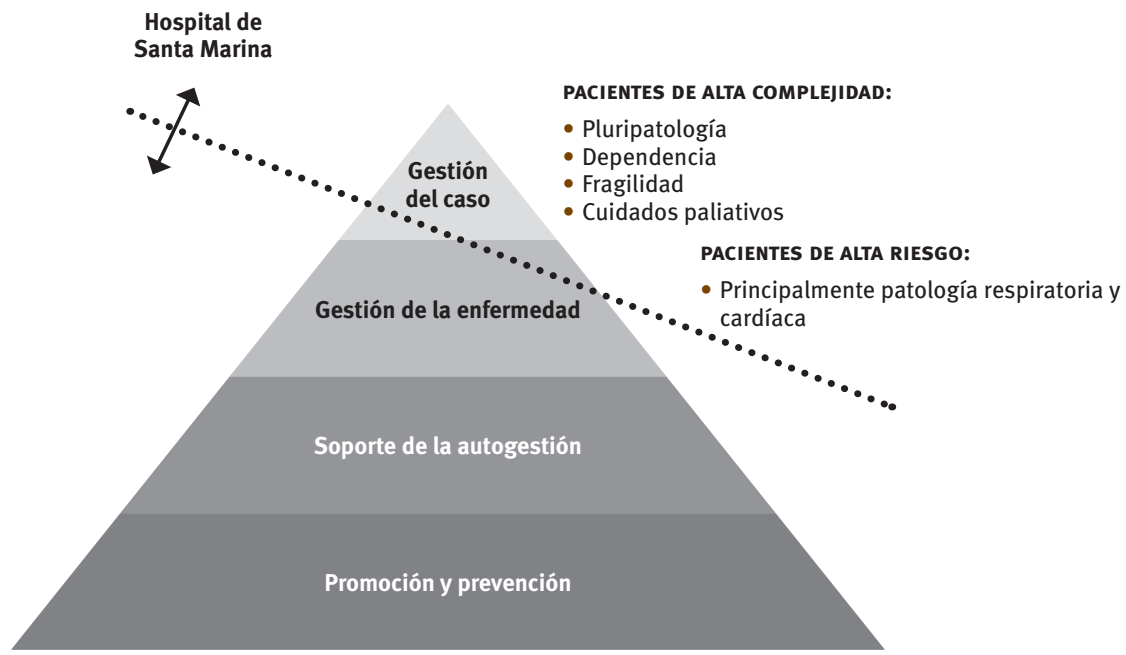

Fuente: Elaboración propia a partir del modelo de riesgos estratificados desarrollado por kaiserpermanente.org en Estados Unidos. 
Si se analiza desde un punto de vista cuantitativo, obtenemos los siguientes resultados económicos del hospital (véase la Tabla 1).

\section{Monetización del valor social: el caso del Hospital de Santa Marina}

El proceso del análisis del valor monetario generado por el Hospital Santa Marina, se inicia con la formación de un equipo de trabajo mixto, formado por profesores de la Universidad de Deusto y de la Universidad del País Vasco (UPV/ EHU) y personas del equipo directivo del hospital (gerente y director económico-financiero). En concreto, en 2017 estaba formado por un equipo de cinco personas, si bien posteriormente se incorpora un profesional más.

La primera fase del trabajo se centró en la elaboración del mapa de stakeholders desde la perspectiva del valor generado. Este mapa, en vez de exponer de forma estratégica a qué stakeholders se dirige la organización, muestra a quién le está generado valor. Es un cambio importante, aunque, obviamente, lo ideal sería que ambas perspectivas estuvieran totalmente alineadas. Este análisis del mapa solo es posible hacerlo con tiempo y a partir de comprobar qué resultados se han ido obteniendo (Figura 2).

Tabla 1. Datos económicos del Hospital Santa Marina 2014-2018 (cifras en euros)

\begin{tabular}{|l|c|c|c|c|c|}
\hline Concepto & $\mathbf{2 0 1 4}$ & $\mathbf{2 0 1 5}$ & $\mathbf{2 0 1 6}$ & $\mathbf{2 0 1 7}$ & $\mathbf{2 0 1 8}$ \\
\hline Gastos generados & 22.992 .249 & 23.467 .654 & 23.431 .566 & 24.216 .411 & 24.650 .634 \\
\hline Gastos de funcionamiento & 3.033 .173 & 3.033 .761 & 2.872 .318 & 2.963 .790 & 3.104 .474 \\
\hline Gastos de personal & 19.959 .076 & 20.433 .893 & 20.559 .249 & 21.252 .620 & 21.546 .160 \\
\hline $\begin{array}{l}\text { \% Trabajadores que tributan en la Diputación Foral de } \\
\text { Bizkaia }\end{array}$ & $92,84 \%$ & $92,97 \%$ & - & - & - \\
\hline Ingresos & 22.867 .421 & 23.416 .915 & 23.490 .462 & 23.890 .120 & 24.652 .567 \\
\hline Resultados del ejercicio & -124.828 & -50.739 & 58.896 & -326.291 & 1.933 \\
\hline Inversiones estratégicas & 151.815 & 256.085 & 0 & 0 & 410.327 \\
\hline Reformas propias & 125.838 & 194.917 & 227.290 & 0 & 542.986 \\
\hline Coste por estancia & 397 & 378 & 448 & 476 & 472 \\
\hline Coste por ingreso & 4.566 & 4.044 & 4.681 & 5.093 & 5.003 \\
\hline
\end{tabular}

Fuente: Elaboración propia.

Figura 2. Mapa de 'stakeholders'

GRUPOS DE INTERÉS: HOSPITAL SANTA MARINA

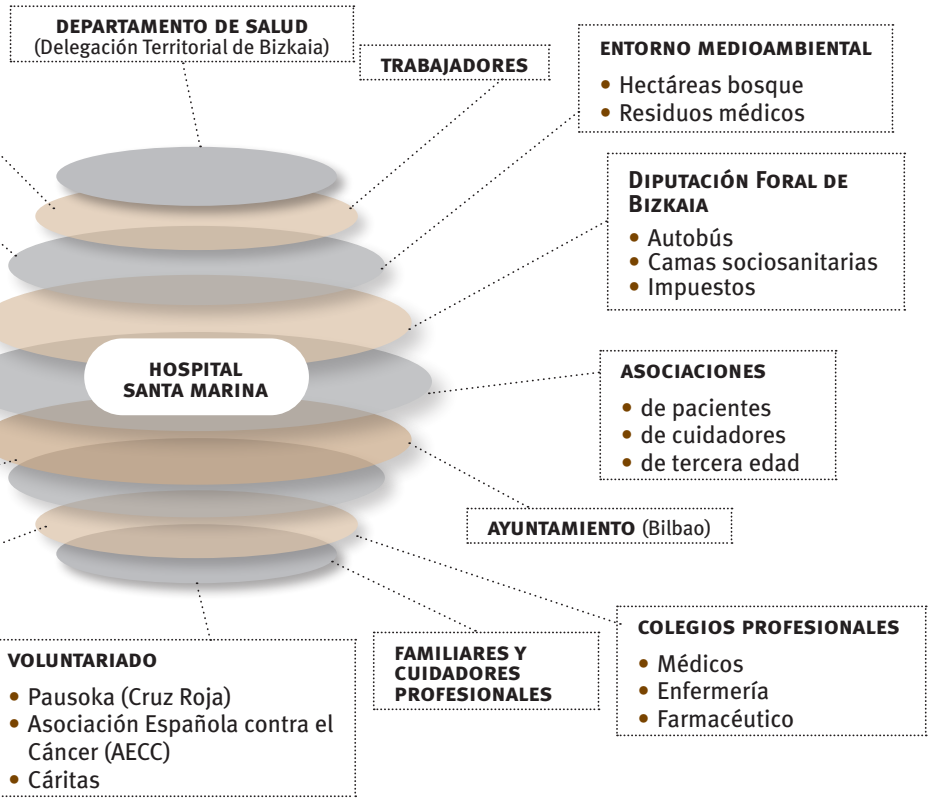

Fuente: Elaboración propia. 
El mapa queda formado por cada uno de los grupos de interés que aparecen en la figura:

1. Pacientes: son la razón de ser del hospital. La mayor parte de los pacientes son personas de edad avanzada, con patología crónica compleja, con quienes hay que trabajar desde la doble vertiente de la cura y el cuidado.

2. Familiares de pacientes: acompañan y cuidan a sus familiares, amigos o allegados.

3. Trabajadores: se encargan del buen funcionamiento en el ámbito global del hospital.

4. Proveedores: abastecen al hospital con los productos o servicios necesarios.

5. Administraciones Públicas: este grupo lo componen la Diputación Foral de Bizkaia, el Ayuntamiento de Bilbao, el Departamento de Salud y otras organizaciones.

6. Cuidadores profesionales: personas que tienen como objetivo acompañar y cuidar a los pacientes y son contratadas por los pacientes o familias.

7. Estudiantes pre/postgrado: el Hospital es proveedor de prácticas de grados técnicos y estudios universitarios.

8. Entorno medioambiental: el entorno medioambiental, aunque es difícil de personalizar como stakeholder, en múltiples trabajos recibe esta categorización. En nuestro caso, hemos identificado las variables de valor para el entorno medioambiental a través del resto de stakeholders; en este caso, actúa como shapeholder (Retolaza, San-José y Aguado, 2016) del medio ambiente. El hospital cuenta con una amplia zona forestal alrededor de sus instalaciones, la cual genera $\mathrm{O}_{2}$ y fija el $\mathrm{CO}_{2}$; además de su impacto visual positivo. Asimismo, se reciclan los residuos sanitarios.

9. Residencias: existe una colaboración y coordinación entre el Hospital de Santa Marina y estas para atender a los pacientes residenciados. Santa Marina es el centro de referencia para la atención de los pacientes crónicos de edad avanzada residenciados de la comarca de Bilbao y parte de la comarca de Barrualde, que requiere de un hospital de crónicos.

10. Sindicatos: defienden los derechos y los intereses profesionales de los trabajadores del hospital.

11. Colegios profesionales: colegios de médicos/as, farmacéuticos/as, enfermeras/os, etc.

Con base en el mismo mapa de stakeholders, se identificó una serie de entidades participantes en los grupos de interés para dialogar con ellas sobre el valor social percibido en relación al Hospital de Santa Marina; asimismo, se seleccionó a las personas de referencia para mantener una entrevista con ellas.

Siendo el objetivo el cálculo del valor social del Hospital de Santa Marina, en primer lugar se calculó el valor social generado por la actividad de mercado de la entidad; en segundo, el valor social de no mercado. El sumatorio consolidado de ambas conforma el valor social integral.

\subsection{Valor socioeconómico directo}

Constituye el impacto económico que crea la organización en uno o varios territorios por la actividad económica. Estos valores pueden ser tanto directos como indirectos. El primero puede definirse como la suma de salarios, seguridad social, valor añadido, impuesto de la renta, IVA, otros impuestos y el resultado del ejercicio (véase la Tabla 2).

Tabla 2. Valor socioeconómico del Hospital de Santa Marina en 2017

\begin{tabular}{|c|c|c|c|}
\hline Descripción & Indicador & Fuente & Resultado \\
\hline Valor añadido & $\begin{array}{l}\text { - valor } \\
\text { añadido } \\
\text { anual }\end{array}$ & Contabilidad & $o €$ \\
\hline Salarios & $\begin{array}{l}\text { - sueldos } \\
\text { netos }\end{array}$ & 10T & $13.588 .369 €$ \\
\hline $\begin{array}{l}\text { Seguridad } \\
\text { Social }\end{array}$ & $\begin{array}{l}\text { - SS } \\
\text { empresa } \\
\text { + s.s. } \\
\text { trabajador }\end{array}$ & Contabilidad & $5.533 .120 €$ \\
\hline $\begin{array}{l}\text { Impuesto sobre } \\
\text { la Renta }\end{array}$ & $\begin{array}{l}\text { - (retención } \\
\text { IRPF) }\end{array}$ & 10T & $2.874 .523 €$ \\
\hline $\begin{array}{l}\text { Otros } \\
\text { impuestos }\end{array}$ & $\begin{array}{c}\text { - impuestos } \\
\text { abonados }\end{array}$ & Contabilidad & o€ \\
\hline Resultado & & Contabilidad & $-326.444 €$ \\
\hline Amortizaciones & & $\begin{array}{l}\text { Contabilidad + } \\
\text { Acuerdo junta }\end{array}$ & $479.384 €$ \\
\hline Inversión & $\begin{array}{l}- \text { fondos } \\
\text { reserva }\end{array}$ & $\begin{array}{l}\text { Contabilidad + } \\
\text { Acuerdo junta }\end{array}$ & $846.296 €$ \\
\hline IVA & $\begin{array}{l}\text { - IVA } \\
\text { devengado }\end{array}$ & $\begin{array}{l}\text { Cálculo a } \\
\text { partir de las } \\
\text { facturas }\end{array}$ & $625.864 €$ \\
\hline
\end{tabular}

En la Tabla 2 se recogen los datos más significativos de la actividad económica, los cuales nos sirven para calcular con posterioridad el Valor Social Directo, y el inducido, en sus dos dimensiones: gasto corriente e inversión.

\section{R-VES $8.407 .642 €$ \\ VES $\mathbf{2 1 . 6 6 9 . 5 6 7 € ~}$}

Junto con el Valor Social generado para cada categoría - valor socioeconómico (VES) - que es la suma del salario neto más las retenciones de IRPF, las cotizaciones a la seguridad social y el resultado del ejercicio del hospital, se calcula la generación de flujos de caja que, de forma directa, indirecta, o como ahorro, son percibidos por la Administración (R-VES), en este caso cotizaciones a la seguridad social y retenciones de IRPF. Así pues, el Hospital de Santa Marina, a través de su actividad mercantil, generó en 2016 un valor para la sociedad de 21.669.567 de euros, y un retorno a la Administración de 8.407.642 de euros. 


\subsection{Valor socioeconómico indirecto: proveedores}

El valor socioeconómico indirecto se refiere al impacto que el hospital genera a otras organizaciones y de forma indirecta al conjunto de la sociedad.

Según el trabajo realizado, el valor social generado de forma indirecta por la compra a proveedores es el que refleja la Tabla 3.

Tabla 3. Valor inducido a proveedores del Hospital de Santa Marina en 2017 (en euros)

\begin{tabular}{|c|c|c|}
\hline \multirow{9}{*}{$\begin{array}{l}\text { ㅁ } \\
\text { 은 }\end{array}$} & Descripción & Resultado \\
\hline & Compra proveedores & $2.759 .870,88$ \\
\hline & Gastos personal & $566.889,66$ \\
\hline & Resultados & $97.561,84$ \\
\hline & Impuestos & $19 \cdot 359,32$ \\
\hline & Seguridad social & $209.749,17$ \\
\hline & Impuesto sobre la renta & $75.835,49$ \\
\hline & IVA & $478.814,93$ \\
\hline & Valor añadido & $809.529,23$ \\
\hline
\end{tabular}

El valor generado de forma indirecta, mediante su efecto tractor, al conjunto de la sociedad asciende, en el año 2017, a 1.288.344, con un retorno a las diversas administraciones públicas de 783.759 .

\subsection{Valor socioeconómico indirecto: proveedores de inversión}

El mismo resultado aplicado al valor indirecto generado a través de los proveedores de inversión, arroja las cifras que se muestran en la Tabla 4.

Tabla 4. Valor a proveedores de inversión del Hospital de Santa Marina en 2017 (en euros)

\begin{tabular}{|c|c|c|}
\hline \multirow{9}{*}{$\begin{array}{l}\bar{\pi} \\
\text { 음 }\end{array}$} & Descripción & Resultado \\
\hline & Compra proveedores & 846.296 \\
\hline & Gastos personal & 217.797 \\
\hline & Resultados & 83.044 \\
\hline & Impuestos & 13.965 \\
\hline & Seguridad social & 80.585 \\
\hline & Impuesto sobre la renta & 28.314 \\
\hline & IVA & 38.787 \\
\hline & Valor añadido & 328.236 \\
\hline
\end{tabular}

El valor global generado por la inversión se calcula sobre los datos de la Tabla 4, con una distribución similar a la de los proveedores de explotación. Los resultados obtenidos ascienden a 367.023 euros en lo relativo al valor social generado, y 161.650 euros de ingreso a la Administración.

\subsection{Valor social de no mercado}

Este apartado es el más relevante y novedoso del estudio, ya que en él se valora de forma objetiva la percepción que tienen los stakeholders del valor que les genera el Hospital de Santa Marina. Como consecuencia de un gran número de entrevistas llevadas a cabo con representantes de todos los stakeholders definidos por el hospital (anteriormente reflejados en su mapa) se ha obtenido una serie de variables a cuantificar, muchas de las cuales no son objetivamente cuantificables, por lo que se han descartado, pero las que sí lo han sido se han valorado en la Tabla 5, y como consecuencia de ello, se ha obtenido que el Hospital de Santa Marina genera un valor percibido por sus stakeholders de 21.725.168 euros.

\subsection{Valor social integrado (VSI)}

La Tabla 6 integra el valor social generado a través de la actividad de mercado (23.324.933 euros), con el valor social específico (21.725.168 euros) ascendiendo el valor social integral (consolidado) a la cantidad de 45.050.101 euros. Si tenemos en cuenta el valor emocional, está cantidad asciende a 55.434.731 euros. El valor emocional se ha calculado de la siguiente manera:

Se ha entendido que si el hospital obtenía un 5 en la valoración de las encuestas de satisfacción de usuarios (rango o a 10) significaba que su aportación a estos era nula, siendo positivas las valoraciones por encima de ese cinco y negativas las inferiores a él. Dado que Santa Marina ha obtenido una valoración media de 9,78, tiene una valoración positiva de 4,78 puntos. En consecuencia, si el valor social especifico de 21.725.168 lo multiplicamos por 0,478 , nos sale 10.384 .630 euros, que sumados a los 45.050.101 euros de valor integral nos da el resultado de 55.434.731 euros de valor socioemocional.

En relación con el retorno socioeconómico sobre la financiación recibida (SROI.E), este es del 0,964 (23.324.933 euros). Por su parte, el valor social generado en relación con la financiación recibida (SROI.S) es de 0,898, y el retorno del valor social integral (SROI.I), asciende a 1,863, llegando al 2,292 si se tiene en cuenta el retorno socioemocional (SEROI).

Por su parte, el flujo de ingresos a la Administración asciende a 9.353.051 euros, siendo el montante de la financiación recibida del contrato programa con el Departamento de Salud de 24.184.002 euros, con un retorno económico sobre la financiación pública (ROI) del 0,387 (-14.830.951 euros) para la Administración (véase la Tabla 7). 
Tabla 5. Valor Social de no mercado en el Hospital de Santa Marina en 2017

\begin{tabular}{|c|c|c|c|c|c|c|}
\hline № & Variables & Indicadores & Output & Valor de referencia & $\begin{array}{c}\text { Proxy } \\
\text { (en euros) }\end{array}$ & $\begin{array}{l}\text { Valor social } \\
\text { (en euros) }\end{array}$ \\
\hline 1 & Hospitalización & № estancias & 41.146 & $\begin{array}{l}\text { Coste medio cama hospital } \\
\text { Osakidetza }\end{array}$ & 384,00 & $15.800 .064,00$ \\
\hline 2 & Hospitalización & № estancias paliativos & 9.116 & $\begin{array}{l}\text { Coste medio cama hospital } \\
\text { Osakidetza }\end{array}$ & 270,00 & $2.461 .320,00$ \\
\hline 3 & Atención urgencias & Urgencias atendidas & 5.664 & $\begin{array}{l}\text { Coste atención medica } \\
\text { urgencias (pública/privada). }\end{array}$ & 153,00 & $866.592,00$ \\
\hline 4 & $\begin{array}{l}\text { Liberar camas en otros } \\
\text { hospitales }\end{array}$ & $\begin{array}{l}\text { Pacientes procedentes de otros } \\
\text { hospitales }\end{array}$ & 20.658 & & 44,85 & $926.511,30$ \\
\hline 5 & $\begin{array}{l}\text { Prácticas reales para } \\
\text { alumnos }\end{array}$ & $\begin{array}{l}\text { № horas curso académico } \\
\text { alumnos en prácticas }\end{array}$ & 4.872 & & 18,00 & $87.696,00$ \\
\hline 6 & Parking gratuito & $\begin{array}{l}\text { № visitantes año / estancia } \\
\text { media }\end{array}$ & 10.039 & OTA / parking & 3,10 & $311.624,40$ \\
\hline 7 & Liberación cuidador & $\begin{array}{l}\text { Estancias de pacientes no } \\
\text { residenciados }\left(30 \%{ }^{\star}\right)\end{array}$ & 16.798 & $\begin{array}{l}10 \text { euros/hora y } \\
\text { una jornada de } 8 \mathrm{~h}\end{array}$ & 40,00 & $401.560,00$ \\
\hline 8 & Apoyo a residencias & $\begin{array}{l}\text { Estancias de pacientes cuyo } \\
\text { origen es residencias }\end{array}$ & 16.798 & & 50,00 & $839.900,00$ \\
\hline 9 & Ahorro emisión & 0 & 23 & $\begin{array}{l}\text { Fijación C02. Derechos por } \\
\text { hectárea }\end{array}$ & $1.300,00$ & $29.900,00$ \\
\hline & & & & \multicolumn{2}{|l|}{ Valor social específico total } & $21.725 .167,70$ \\
\hline
\end{tabular}

(*) Dato facilitado por la asistencia social del Hospital

Tabla 6. Valor Social Integrado del Hospital de Santa Marina en 2017

\begin{tabular}{|c|c|c|c|c|}
\hline \multirow{2}{*}{ Variables } & $\begin{array}{l}\text { Valor económico social } \\
\text { (en euros) }\end{array}$ & $\begin{array}{l}\text { Valor social específico } \\
\text { (en euros) }\end{array}$ & $\begin{array}{l}\text { Valor social integral } \\
\text { (en euros) }\end{array}$ & \multirow{2}{*}{ Acrónimos } \\
\hline & VES & VASE & VASI & \\
\hline Impacto directo de la actividad económica & 21.669 .567 & & 21.669 .567 & VES \\
\hline Impacto indirecto, proveedores & 1.288 .344 & & 1.288 .344 & VES-IP \\
\hline Impacto indirecto, proveedores de inversión & 367.023 & & 367.023 & VES-IP.I \\
\hline Valor social específico & & 21.725 .168 & 21.725 .168 & VSE-F \\
\hline Valor integral bruto & 23.324 .933 & 21.725 .168 & 45.050 .101 & VASI-Bruto \\
\hline Financiación publica & 24.184 .002 & 24.184 .002 & 24.184 .002 & \\
\hline Valor integral neto & -859.069 & -2.458 .834 & 20.866 .099 & VASI-Neto \\
\hline Valor socioemocional & & 32.109 .798 & 55.434 .731 & V-EMO \\
\hline Ratio Retorno Caja. Financiación Pública & & & & ROI \\
\hline $\begin{array}{l}\text { Ratio Retorno Económico. Financiación } \\
\text { Púbica }\end{array}$ & \multicolumn{2}{|c|}{0,964} & & SROI.E \\
\hline Ratio Retorno Social. Financiación Pública & & 0,898 & & SROI.S \\
\hline $\begin{array}{l}\text { Rato Retorno Social Integral (Social + } \\
\text { Económico) }\end{array}$ & & & 1,863 & SROI.I \\
\hline Ratio Retorno Socioemocional & & 1,328 & 2,292 & SEROI \\
\hline
\end{tabular}

Tabla 7. Retorno económico a la Administración Pública del Hospital de Santa Marina (en euros)

\begin{tabular}{|l|c|}
\hline $\begin{array}{l}\text { Variables para monetizar el valor social } \\
\text { Perspectiva de la contabilidad social }\end{array}$ & Retorno económico Administraciones Públicas \\
\hline Impacto directo de la actividad económica & 8.407 .642 \\
\hline Impacto indirecto proveedores & 783.759 \\
\hline Impacto indirecto proveedores de inversión & 161.650 \\
\hline Valor integral bruto & 9.353 .051 \\
\hline Financiación pública & 24.184 .002 \\
\hline Valor integral neto & -14.830 .951 \\
\hline Ratio Retorno Caja. Financiación Pública & 0,387 \\
\hline
\end{tabular}

Fuente: Elaboración propia. 
Este resultado se puede entender como que solo 3,87 de cada 10 euros invertidos por la Administración en el hospital retornan a ella por medio de la actividad económica del centro (cotizaciones a la seguridad social y pago de impuestos como retenciones de IRPF e IVA pagado a proveedores). Pero esta es solo una lectura parcial, pues a nuestro entender, caben, al menos, dos conclusiones más:

1. Realmente la Administración invierte a "fondo perdido" en el hospital solo 14.830 .951 euros, ya que de los 24.184.002 euros que en 2017 aportó vía contrato programa, 9.353.051 euros se revierten de nuevo a ella como actividad económica generada.

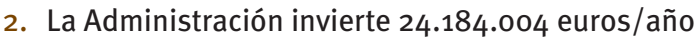
en el hospital, pero este genera un valor anual de 45.050.101 euros sin contar el valor emocional. Es decir, el hospital prácticamente duplica el valor generado sobre la inversión en él realizada por los poderes públicos.

En la siguiente figura (véase la Figura 3) se refleja de forma sucinta el valor social generado por el Hospital de Santa Marina, para los diversos ecosistemas en el año 2017. El hospital recibe de la Administración 24,2 millones de euros y genera un valor social de 55,4 millones de euros, por lo que revierte 2,29 veces lo que recibe.

\section{Evolución: periodo 2014-2018}

La evolución de los valores obtenidos por el hospital en los años 2014 a 2018 se puede apreciar en la Tabla 8.

Dado que no se ha hecho más que una entrevista a los stakeholders y que el presupuesto anual del hospital es muy estable, era de esperar una escasa evolución de los resultados interanuales, confirmándose que por cada euro recibido el hospital genera más de dos. No obstante, la evolución es claramente decreciente, por lo que el hospital debe plantearse su enfoque estratégico si pretende ser referente en la generación de valor social.

Por otro lado, es muy importante resaltar que la contabilidad financiera en sí misma puede no reflejar el verdadero valor generado de una organización. Si observamos el resultado del ejercicio de los cinco años, vemos que este siempre está cercano a

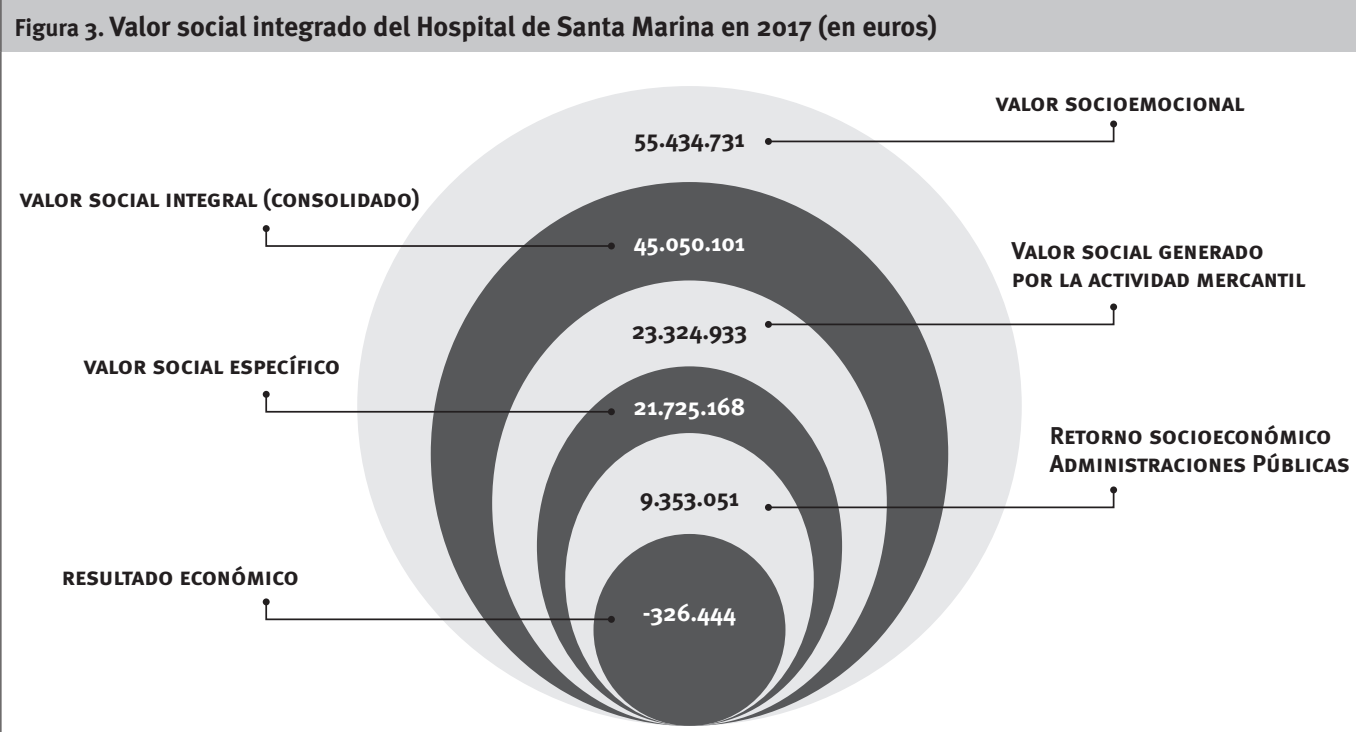

Fuente: Elaboración propia.

Tabla 8. Evolución del periodo 2014 al 2018 (en euros)

\begin{tabular}{|c|c|c|c|c|c|}
\hline & 2014 & 2015 & 2016 & 2017 & 2018 \\
\hline Resultado económico & -24.828 & -50.739 & 55.896 & -326.444 & -280.759 \\
\hline $\begin{array}{l}\text { Retorno socioeconomico } \\
\text { Administraciones Públicas }\end{array}$ & 8.501 .186 & 8.994 .364 & 9.059 .853 & 9.353 .051 & 7.492 .900 \\
\hline Valor social act. mercantil & 21.501 .031 & 22.551 .648 & 22.941 .889 & 23.324 .933 & $19.974 \cdot 343$ \\
\hline Valor social específico & 24.639 .000 & 23.745 .496 & 22.228 .295 & 21.725 .168 & 22.668 .295 \\
\hline Valor social integral & 46.140 .031 & 46.297 .144 & 45.170 .184 & 45.050 .101 & 42.642 .638 \\
\hline Valor socioemocional & 58.336 .336 & 57.766 .219 & 55.684 .167 & $55 \cdot 434.731$ & 51.256 .590 \\
\hline
\end{tabular}

Fuente: Elaboración propia a partir de San-José et al. (2019). 
cero. Esto es debido a que el centro cuenta con un presupuesto de ingresos y gastos muy rígido en el que tiene escaso margen de maniobra; por tanto, su cuenta de pérdidas y ganancias difícilmente reflejará adecuadamente sus procesos de gestión. Asimismo, este método novedoso de análisis (valor social generado para los grupos de interés), puede permitir a los gestores expresar un valor diferente de su organización que el que refleja la cuenta de pérdidas y ganancias. Esto puede tener especial relevancia en la gestión pública, donde la cuenta de resultados, en muchas ocasiones, tiende a cero (gastos = ingresos), y por tanto puede ser un buen punto de apoyo para la gestión pública que tantas veces es minusvalorada.

\section{Discusión, reflexiones y aplicabilidad de la contabilidad social en otros hospitales}

Con objeto de contribuir no solo a la reflexión académica, sino también a futuras aplicaciones de la contabilidad social en el sector sanitario, se han realizado una serie de reflexiones junto con los responsables del hospital. Así, a continuación, se exponen varias perspectivas que podrán resultar útiles al sector sanitario cuando decidan realizar aplicaciones y análisis del valor social que generan. De antemano, hay que recordar que el propio hecho de realizar un proceso de esta índole, inédito en el área, evidencia su interés para los directores de hospital. Por otro lado, cabe recalcar que, si bien el número final es de interés, el propio proceso y el sistema de reflexión permite lograr sinergias, realizar análisis y comunicar el interés y preocupación del hospital hacia sus stakeholders.

En definitiva, lo esencial de aplicar este proceso no puede ser el resultado, porque si no, estaríamos una vez más analizando las organizaciones desde su finalidad instrumental; en este caso el medio será el fin y lo que enriquecerá a la organización; eso y el uso que luego se dé a toda esta reflexión.

Así, se han realizado reflexiones en tres líneas. La primera desde una perspectiva ex ante o durante el proceso de ejecución; la segunda, el significado que el proceso ha tenido para el Hospital de Santa Marina, y por último una perspectiva ex post con objeto de aprovechar este trabajo para poder transmitir y trasladar a otros hospitales esta contabilidad social.

\subsection{Dificultades durante el proceso de monetización del valor social del Hospital de Santa Marina}

El proceso en sí mismo es lo que genera valor. No obstante, es importante no solo que el responsable lo interiorice, sino que también el resto del equipo lo comprenda y, además de saber de su existencia, lo considere parte de las acciones del hospital. Así que en este sentido transmitir al equipo directivo el interés por aplicar una técnica novedosa e inédita en los hospitales de medición de valor social no fue tarea sencilla. Lo innovador no es, en general, recibido de forma entusiasta y el miedo hacia lo desconocido a veces hace que nos paralicemos. En palabras del director financiero, "ser capaz de trasmitir el interés que podía tener para un centro hospitalario pequeño como el nuestro e intentar cuantificar el valor percibido por nuestros grupos de interés fue una de las tareas más complicadas”.

Otra de las principales dificultades fue el hecho de tener que replantear el mapa de stakeholders desde el valor percibido y no exclusivamente desde el proceso estratégico de la organización. Esta labor y reflexión, aunque teóricamente es sencilla, para algunas organizaciones es complejo pasar de pensar "a quién quiero generar valor" a "a quién genero valor”. Además, la reflexión suele generar el replanteamiento de otro punto de vista que hasta el momento no era central. Como positivo, se puede señalar que esto permite cambiar la perspectiva; se cambia y la centralidad la tienen los stakeholders. Es un gran reto y lograrlo no es siempre tarea fácil.

Por último, la comunicación de los resultados a los stakeholders (en especial a los trabajadores) tampoco fue tarea fácil; de hecho, es una de las partes que se considera desde la dirección del hospital que debería mejorar: “El proceso es excelente, los implicados lo interiorizan, pero no creo que fueran capaces de transmitir la importancia que tuvo para el hospital en su conjunto; al menos, los trabajadores no lo han comprendido de la misma forma. Posiblemente, sea necesario ser parte de un proceso para poder sentirse partícipe de él y poder hacerlo propio, y así comprender el valor social que realmente tiene un hospital".

\subsection{Aspectos gratificantes durante el proceso de monetización del valor social del Hospital de Santa Marina}

El director del hospital destacaba que existen múltiples aspectos enriquecedores, entre los que señaló los más significativos. En primer lugar, fueron la cercanía y capacidad de entender las particularidades y necesidades del Hospital de Santa Marina. Sin duda, lo más valorado es poder transformar lo complejo que es el valor social generado en algo sencillo y más impactante aún, monetizado.

En segundo lugar, la propia contabilidad social, que da una visión diferente a la propia de la contabilidad financiera (la de la pura actividad económica), ya que aporta el valor percibido por los grupos de interés. Esto es fundamental en algunos sectores, pero en la situación en la que nos encontramos lo es en todas las organizaciones.

Por último, el sistema refuerza la gestión pública ante los usuarios y ante los políticos, ya que ofrece una visión del "negocio" desde varios puntos de vista, incluso el del retorno para la Administración. 
En palabras del director, "este sistema legitima a la Administración Pública y permite realizar análisis de su eficiencia a futuro".

\subsection{Uso y aplicabilidad del proceso de contabilidad social}

En cuanto al uso y la aplicabilidad de esta contabilidad social que permite conocer qué impacto social ha tenido un hospital en un periodo de tiempo, un año generalmente, cabe destacar al menos dos aspectos en términos generales:

1. El trabajo aporta información muy relevante ya que además de la propia información económico financiera se genera la visión del valor percibido por los grupos de interés o stakeholders en los que se encuentra la propia administración pública incluida. Así, permite ofrecer información sobre los retornos a la administración de la actividad lo que hace que se pueda ver el neto de la aportación pública.

2. La contabilidad social mediante el cálculo del Valor Social Integrado y la aplicación de la metodología SPOLY ${ }^{2}$ permite complementar muy bien la visión que las organizaciones están ofreciendo sobre su responsabilidad social (por ejemplo, mediante el uso de memorias de la Global Reporting Initiative). Asimismo, a los gestores les da un nuevo enfoque de pensamiento sobre lo que sus grupos de interés esperan de ellos por lo que pueden cambiar/ adaptar sus decisiones de gestión. Esto puede ser integrado, precisamente en sus planes de acción y en sus planes estratégicos, con objeto de dar respuesta a los intereses de los stakeholders de forma holística.

En términos particulares, y para el caso que presentamos, destacamos los tres principales aspectos:

1. Permitió cifrar en unidades monetarias (euros) el valor social percibido por los grupos de interés. Esto ha sido muy relevante y útil, ya que se alinea con la contabilidad financiera y permite eliminar la subjetividad de lo social con entidad exclusivamente cualitativa. Es un paso más allá, debido a la transformación de lo cualitativo en cuantitativo, pero no solo eso, ya que se transforma en unidades monetarias. Esto permite análisis globales y holísticos.

2. El proceso de contabilidad está liderado por profesores universitarios, los cuales han conseguido establecer un modelo matemático avalado científicamente, lo que aporta mucha objetividad a los resultados y los puede hacer comparables con otros. Asimismo, una

${ }^{2}$ La metodología SPOLY, más conocida como el modelo poliédrico, es un modelo de monetización del valor social creado por el Grupo de Investigación ECRI de la Universidad del País Vasco (UPV-EHU) y la Universidad de Deusto (San-Jose, Mendizabal y Retolaza, 2020). agrupación les da el soporte para impulsarlo y divulgarlo (GEAccounting).

3. Desde la dirección señalan que este proceso permite visibilizar el valor de lo público, el cual no siempre está adecuadamente reconocido.

\subsection{Recomendaciones y sugerencias para futuros hospital con interés de aplicar la contabilidad social en sus entidades}

Sin duda es posible que otros hospitales puedan aplicar este modelo. La experiencia es muy gratificante y da una visión completamente diferente a lo que los hospitales están acostumbrados en la gestión. Como parte de la Administración Pública, los gestores de hospitales deben ser los primeros interesados en medir el valor social, ya que el carácter de lo público ya les debe predisponer a crear/generar valor social, lo cual no siempre lo entienden así. Sin duda, si es público, debería ser entendido como bueno para la sociedad. En la actualidad, se han comenzado a realizar los primeros pasos para la estandarización, y para ello hay varios proyectos en marcha.

Por destacar algunas últimas reflexiones con objeto de trasladar la contabilidad social a otros hospitales:

1. El proceso en su conjunto se vería mejorado por las aportaciones de otros centros con sus particularidades, lo cual le permitiría ser más objetivo y contrastado.

2. La utilización de la contabilidad social en un mayor número de hospitales permitirá el benchmarking y una mejora colectiva hacia lo social. Sin duda, el proceso se enriquecerá con el análisis de otros hospitales.

\section{Conclusiones, limitaciones y futuras líneas}

Las entidades públicas se deben a la sociedad; de hecho, su existencia viene medida por el nivel de respuesta hacia el logro de cumplir las necesidades que los receptores de la organización tienen. En este sentido, un hospital claramente no tiene las mismas necesidades y obligaciones que una organización empresarial, y mucho menos que la mercantil. Así que los hospitales públicos deben exponer, o deberían exponer, qué valor social están generando. Ciertamente, parte de su valor social viene dado por su actividad económica, la cual se recoge en la contabilidad económico-financiera, pero existen muchas otras actividades y acciones que no se recogen en la contabilidad tradicional porque no suponen transacciones monetarias, aunque no por ello no podemos confirmar que no generen valor. Todo lo contrario.

Para realizar este trabajo se ha seleccionado un hospital, en este caso, el Hospital de Santa 
Marina, de Osakidetza Servicio Vasco de Salud, por un lado, por ser un hospital con una tipología de paciente muy concreta, $y$, por otro lado, por ser un hospital de tamaño medio. Se ha aplicado la contabilidad social con objeto de conocer cuál es el valor social generado. Así, se ha determinado que es posible conocer cuál es el valor social generado por un hospital, y llegar a monetizarlo. El interés de aplicar la contabilidad social denota interés y preocupación de sus directivos en lo social, pero más importante aún, denota interés en mejorarlo. Más aún, este proceso es trasladable a otros hospitales, y aunque aún existen limitaciones, es destacable su aporte hacia lo social, su transformación de lo social en monetario para poder realizar comparaciones holísticas, y su sistematicidad y utilidad en los planes estratégicos a futuro. Sin duda, es uno de los sistemas de información social que más repercusión pueden tener en el futuro para que los aspectos sociales no sean instrumentales sino centrales.

Existen varias limitaciones; una de ellas es el escaso número de hospitales donde se ha aplicado, y otra el hecho de no tener una estandarización que permita un traslado escalable a otros hospitales. Sin embargo, se espera que en un futuro cercano ambas sean futuras líneas de trabajo y así poder, por un lado, aplicar la contabilidad social a un mayor número de hospitales, consensuar variables y proxys comunes, y poder realizar el traslado a un grupo de hospitales para lograr la estandarización del sistema. En ese momento, se logrará una comparabilidad de estas entidades públicas en otros ámbitos que no son el económico, lo que permitirá una amplitud de conocimiento sobre lo que realmente están aportando a la sociedad y al bienestar de sus stakeholders. Sin duda, su razón de ser. 


\section{Bibliografía referenciada}

BERNAL, R.; SAN-JOSÉ, L. y RETOLAZA, J.L. (2019): "Improvement Actions for a More Social and Sustainable Public Procurement: A Delphi Analysis", Sustainability, no 11, 4069: 9; doi:10.3390/su11154069.

EUSTAT (2014): Informe III EUSTAT 2014: Proyecciones de Población 2026 - Análisis de Resultados, (https://www.eustat.eus/estadisticas/ tema_163/opt_o/tipo_3/ti_Proyecciones_de poblacion/temas.html).

GUZMÁN, B.; MENDOZA, J. y PÉREZ, V. (2020): “Informe del valor social del hotel Tigaiga", CIRIECEspaña, revista de economía pública, social y cooperativa, $\mathrm{n}-\mathbf{0} 100$, pendiente publicación.

HARRISON, J. S.; PHILLIPS, R.A., y FREEMAN, R.E. (2020): "On the 2019 Business Roundtable 'Statement on the Purpose of a Corporation'", Journal of Management, 46(7), 1.223-1.237.

LAZCANO, L.; SAN-JOSÉ, L. y RETOLAZA J.L. (2019): “Social Accounting in the Social Economy: A Case Study of Monetizing Social Value", in Santos, C. et al (Eds.), Modernization and Accountability in the Social Economy Sector, IGI Global, pp. 132-151.

LAZKANO, L. y SAN-JOSÉ, L. (2020): “Monetización del valor social: el caso Clade", Gizarte Ekonomiaren Euskal Aldizkaria-Revista Vasca de Economía Social, 16, 103-127, <https://doi.org/10.1387/ reves.20903'.
LAZKANO, L. y BERAZA, A. (2019): "Social Accounting for Sustainability: A Study in the Social Economy", Sustainability, 11 (24), 6.894-20.

RETOLAZA, J. L.; SAN-JOSÉ, L., y AGUADO, R. (2016): “The role of shapeholders as a link between a firm and non-stakeholders: the pursuit of an economy for the common good based on stakeholder theory", en Corporate Responsibility and Stakeholding, pp. 31-48, Emerald Group Publishing Limited.

RETOLAZA, J. L.; SAN-JOSÉ, L. y RUÍZ-ROQUEÑI, M. (2016): Social accounting for sustainability Monetizing the social value. Cham, Springer.

RETOLAZA, J.L.; SAN-JOSÉ, L.; RUIZ-ROQUEÑI, M. y BARRUTIA, J. (2014): "Cuantificación del valor social: propuesta metodológica y aplicación al caso de Lantegi Batuak", Zerbitzuan, 55, 17-33. https://doi.org/10.5569/1134-7147.55.02.

SAN-JOSÉ L.; MENDIZABAL, X. y RETOLAZA, J.L. (2020): "Social accounting and business legitimacy", en RENDTORFF, J. (ed.), Handbook of Business Legitimacy, Springer, 〈https://doi. org/10.1007/978-3-319-68845-9_88-1).

SAN-JOSÉ, L.; RETOLAZA, J.L., y BERNAL, R. (en prensa): Índice de valor social añadido: una propuesta para analizar la eficiencia hospitalaria. Gaceta Sanitaria, https://doi.org/10.1016/j. gaceta.2019.08.011 